\title{
Resolved Hubble space spectroscopy of ultracool binary systems ${ }^{\star, \star \star}$
}

\author{
E. L. Martín ${ }^{1,2}$, W. Brandner ${ }^{3}$, H. Bouy ${ }^{1}$, G. Basri ${ }^{4}$, J. Davis ${ }^{2}$, R. Deshpande ${ }^{2}$, and M. M. Montgomery ${ }^{2}$ \\ 1 Instituto de Astrofísica de Canarias, 38200 La Laguna, Spain \\ e-mail: ege@iac.es \\ 2 University of Central Florida, Dept. of Physics, PO Box 162385, Orlando, FL 32816-2385, USA \\ 3 Max-Planck Institut für Astronomie, Königstuhl 17, 69117 Heidelberg, Germany \\ 4 Astronomy Department, University of California, Berkeley, CA 94720, USA
}

Received 9 September 2005 / Accepted 19 April 2006

\section{ABSTRACT}

\begin{abstract}
Using the low-resolution mode of the Space Telescope Imaging Spectrograph (STIS) aboard the Hubble Space Telescope (HST), we obtain spectra from 20 spatially-resolved, ultracool dwarfs, eighteen of which belong to nine very low-mass binary systems with known angular separations in the range 0'.37-0.'098. For these dwarfs, we derive their spectral types to be in the range dM7.5 to dL6 by either obtaining their PC3 index value or by comparing our STIS spectra with ground-based spectra of similar spectral resolution from Martín et al. (1999b). We have searched for $\mathrm{H}_{\alpha}$ emission in each object, but the emission is clearly detected in only two of them. We find that the distribution of $\mathrm{H}_{\alpha}$ emission in our sample is statistically different from that of single field dwarfs, suggesting an intriguing anti-correlation between chromospheric activity and binarity for M7-M9.5 dwarfs. We provide strength measuments of the main photospheric features. We derive calibrations of spectral subclasses versus $F 814 W$ and $K$-band absolute magnitudes for a subset of 10 dwarfs in 5 binaries that have known trigonometric parallaxes.
\end{abstract}

Key words. stars: low-mass, brown dwarfs - stars: late-type - techniques: spectroscopic - stars: binaries: visual - Galaxy: solar neighbourhood - stars: fundamental parameters

\section{Introduction}

Late-type, nearby, spatially-resolved binaries, usually referred to as visual binaries, have been patiently followed up for many decades to obtain orbital parameters (e.g., van de Kamp 1938; Couteau 1957; Chang 1972; Abt \& Levy 1973). The dynamical masses obtained from the studies of resolved binaries have been used to calibrate the mass-luminosity relationship for low-mass stars (e.g., Delfosse et al. 2000). A number of recent surveys have extended the search for resolved binaries to include very low-mass (VLM) stars and brown dwarfs (BDs). Hubble Space Telescope imaging has been used to search for VLM binaries ${ }^{1}$ in young associations and open clusters (Martín et al. 2000b, 2003; Kraus et al. 2005) and in the solar neighborhood (Martín et al. 1998, 1999b; Reid et al. 2001; Bouy et al. 2003; Burgasser et al. 2003; Gizis et al. 2003; Golimowski et al. 2004). Near infrared imaging on large ground-based telescopes, usually assisted by adaptive optics, has also been effective in finding VLM binaries (e.g., Koerner et al. 1999; Martín et al. 2000a; Close et al. 2003; Potter et al. 2002; Siegler et al. 2005). These studies have determined that the binary frequency is about $15 \%$ among field VLM stars and BDs in the separation range 1-15 AU, and a sharp drop in the binary frequency is seen for separations larger than 15 AU. Nevertheless, a few examples of wider-field,

* Based on observations obtained with the NASA/ESA Hubble Space Telescope.

$\star \star$ Tables 1-5 and Figs. 3-12 are only available in electronic form at http://www.edpsciences.org

1 In this paper we adopt the definition that a VLM binary has a primary with spectral type M6 and later. ultracool binaries are known (Martín et al. 1998, 2000b; Billères et al. 2005; Phan Bao et al. 2005; Burgasser \& McElwain 2006). On the other hand, the VLM binary frequency is not well established within $1 \mathrm{AU}$ because of lack in long term, spectroscopic binary surveys for VLM stars and BDs. The statistical properties of VLM binaries will continue to be investigated because they provide a fundamental constraint for models of VLM star formation (Kroupa et al. 2003; Umbreit et al. 2005).

As the number of VLM binaries increases, follow-up and statistical studies can then be made to establish the binaries' properties. Astrometric monitoring of three VLM binaries have yielded the first estimates of the orbital parameters and dynamical masses (Lane et al. 2001; Bouy et al. 2004; Brandner et al. 2004). Spatially-resolved, low-resolution, spectroscopic followup studies have resulted in estimates of spectral types (Lane et al. 2001; Goto et al. 2002; Potter et al. 2002; Bouy et al. 2004; Chauvin et al. 2004; Luhman 2004, 2005; McCaughrean et al. 2004; Billeres et al. 2005; Burgasser \& McElwain 2006). Resolved, high-resolution, spectroscopic observations have been reported for only one VLM binary, namely GJ 569 B, thus allowing for dynamical estimates of individual masses to be found (Zapatero Osorio et al. 2004; Simon et al. 2006). Accurate dynamical masses for an eclipsing BD binary have also been reported recently (Stassun et al. 2006).

In this paper, we present resolved, low-resolution, spectroscopic observations of nine VLM binaries, thereby significantly increasing the number of members of VLM systems that have been characterized spectroscopically. In Sect. 2, we describe the selection of the sample, the STIS observations and the processing of the data. In Sect. 3 we describe the spectra, we present the 

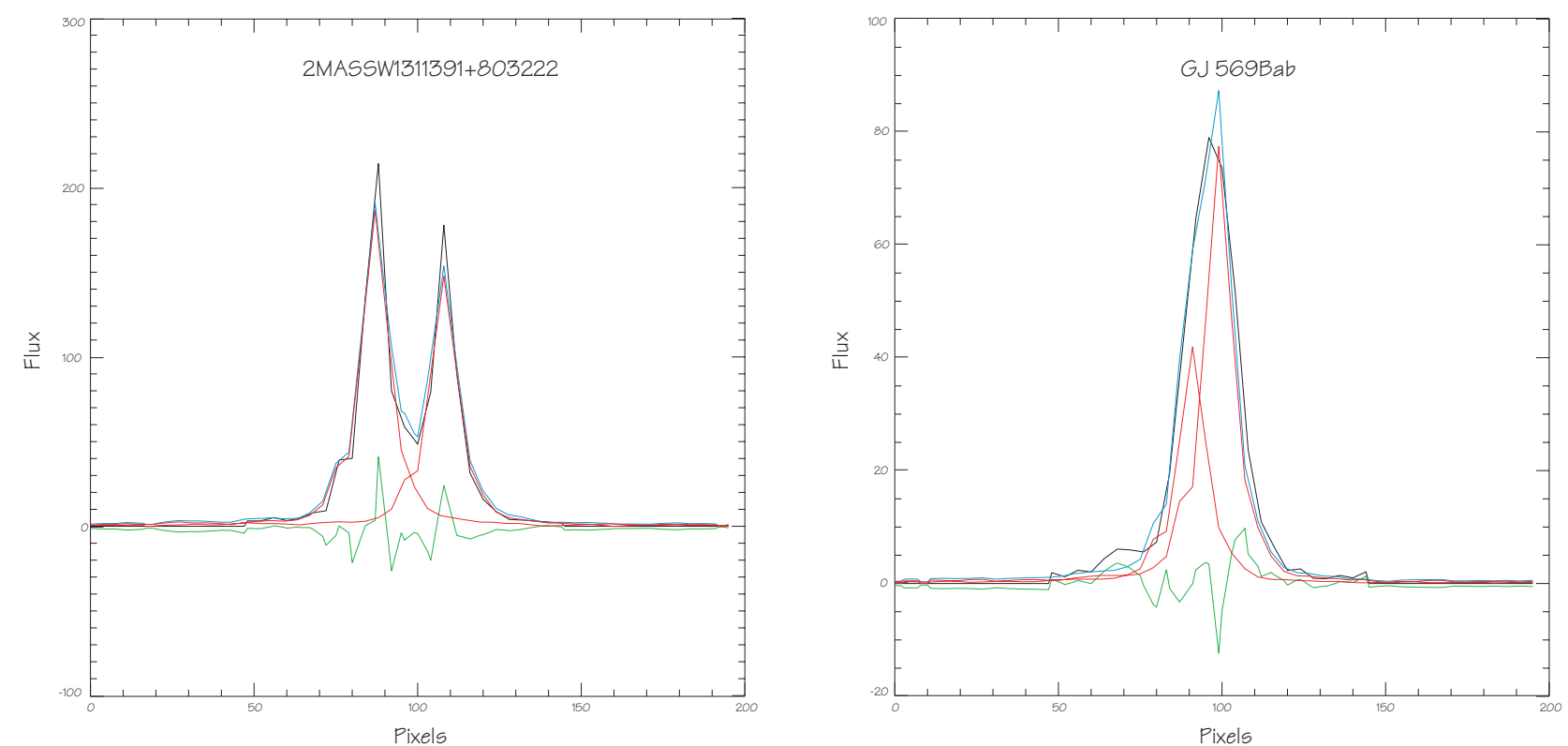

Fig. 1. This figure shows the PSF fitting to the 2-dimensional STIS spectrum at $743.8 \mathrm{~nm}$ for two binary systems, namely 2MASSW J1311391+803222 (separation = 0'.263) and GJ 569 B (separation = 0'090). The black lines show the observed profiles, the red lines indicate the best PSF fits for the components of these systems, the light blue line denotes the sum of the best PSF fits, and the green line shows the residuals obtained by substracting the sum of the PSF fits to the observed profile. The integrated flux of the residuals is about $3 \%$ of the integrated flux of the total PSF.

methods used to determine the spectral types. In Sect. 4, we discuss the chromospheric activity and the main absorption features in our sample, and we derive calibrations of spectral subclass versus absolute magnitudes for the components of VLM binaries with known parallaxes. Finally, in Sect. 5, we summarize our results.

\section{Observations and data processing}

Our sample is selected from known VLM binaries with separations in the range 0.37-0.'098 and I-band magnitude brighter than $I=19$, so that resolved, optical, low-resolution spectroscopy of each component could be obtained with STIS. Table 1 provides the STIS observing log. The observations of 2MASSW J07464256+2000321 have already been presented in Bouy et al. (2004), but we also include them in our analysis for completeness.

Our goal is to obtain spatially-resolved spectra of each component of the binary systems. However, scheduling constraints of HST did not allow for perfect slit alignment along the axis of each binary, making the goal difficult to achieve. For one binary, namely DENIS-P J144137.3-094559, we could not get any spectrum of the secondary because the slit was not oriented along the binary semimajor axis. Another binary, namely 2MASSW J0920122+351742, could not be resolved at the epoch of observations. We note that this object may not be a binary because it has been resolved in only one epoch, but not in other epochs (Bouy et al. 2006, in preparation). For the other binaries, we obtain resolved, 2D spectra and perform the extraction of the two spectra as explained in detail in Bouy et al. (2004). An example of the de-blending of spectra in two of our binary systems (including one of the tightest) is shown in Fig. 1. We use the bias, flat field, and wavelength calibrations that are provided by the HST pipeline.

The grating used is the G750L centered at $775.1 \mathrm{~nm}$, and the aperture is $52^{\prime \prime} 0$ long by 0.2 wide. The total wavelength range of each spectrum is $525-1100 \mathrm{~nm}$, but the usable range depends on the brightness and the spectral type of each object. Residual fringing is clearly present at wavelengths longer than about $900 \mathrm{~nm}$. The nominal dispersion is $4.92 \AA /$ pixel. According to the STIS handbook, our slit width is expected to project onto 4 pixels $(F W H M=20 \AA$ ). For comparison, the resolution quoted by Kirkpatrick et al. (1999) is $9 \AA$, and the resolution of the spectra presented in Martín et al. (1999b, hereafter M99) is $12 \AA$. Thus, our STIS data have a resolution somewhat lower (but comparable) than those papers. In Fig. 2, we display final spectra for three of our targets spanning the whole range of spectral subclasses in our sample.

\section{Spectral classification}

Our resolved, STIS spectra of VLM binaries allow us to determine the spectral type of each component. Only two of our program binaries have previous determinations of spectral type for each component: Lane et al. (2001) gave spectral types of M 8.5 and M 9 for GJ $569 \mathrm{Ba}$ and $\mathrm{Bb}$, respectively, using low-resolution, near-infrared ( $J$-band) spectra. Bouy et al. (2004) obtain spectral types of dL0 and dL1.5 for 2MASSW J0746425+200032 A and B, respectively, using the same STIS data as this work. They compare the STIS spectra with ground-based, low-resolution spectra from M99.

In this work, we estimate the spectral subclasses using two methods: (a) we measure the PC3 index as defined in M99 and then use the PC3-spectral type relationship for $M$ and L dwarfs, and (b) we compare our spectra with those of M99 and choose the best match. The spectral subclasses obtained from both approaches are given in Table 2 . When the subclasses from the two methods agree within one subclass, we compute the average and then round in steps of 0.5 subclass. When the two methods disagree, we favor the subclass derived from method (b) because it includes more spectral information. This disagreement is mainly seen in the latest $\mathrm{L}$ dwarfs of our sample. 


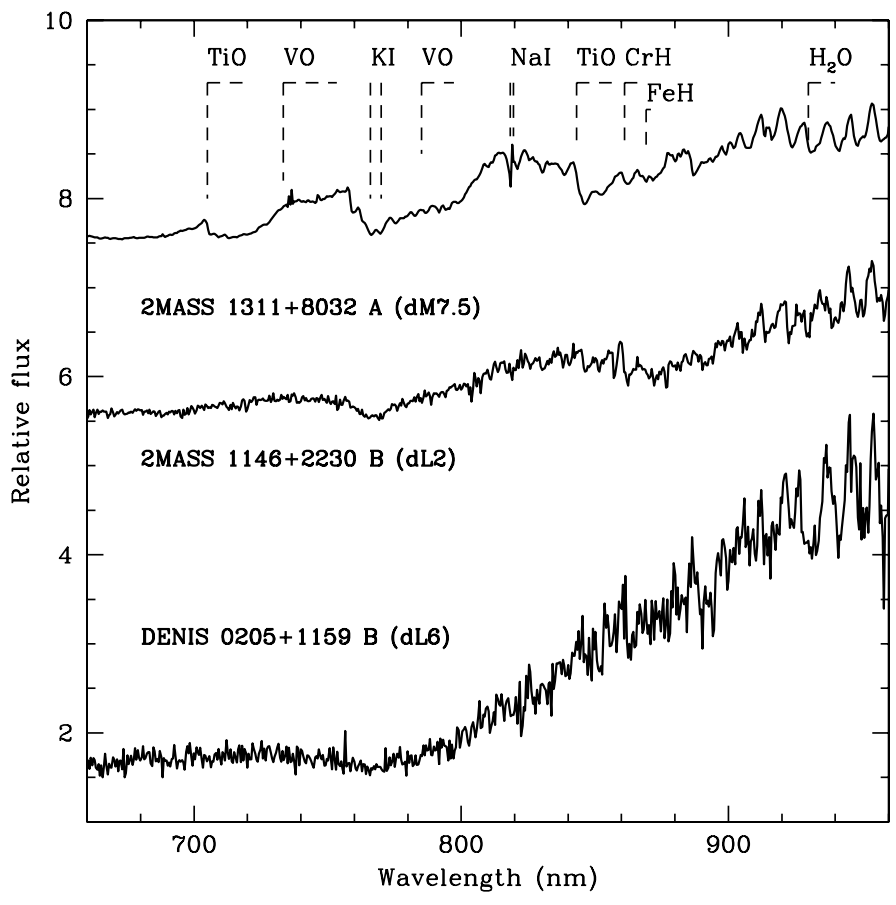

Fig. 2. Final STIS spectra of three resolved ultracool dwarf binaries covering a representative range of spectral subclasses. The main spectral features identified in these spectra are labelled.

\subsection{Description of the spectra of each binary}

In this subsection, the STIS spectra of each target are displayed and then they are compared with the best matching M99 spectra:

- DENIS-P J020529.0-115925 A (dL5): the K I resonance doublet is slightly broader, and the $\mathrm{CrH}$ and $\mathrm{FeH}$ bands are weaker than in the M99 spectrum of DENIS-P J1228-2415 (Fig. 3). The M99 spectrum of DENIS-P J0205-1159 is the best match to our STIS spectrum of DENIS-P J020529.0-115925 A, indicating, as expected, that the primary dominates the composite ground-based spectrum. We note that the PC3 index method gives a slightly earlier subclass, namely dL4.1, than the spectrum matching technique.

- DENIS-P J020529.0-115925 B (dL6): the CrH and FeH bands have almost vanished. The M99 spectrum of DENIS-P J0255-4700 is the best match except for the steam band at $930 \mathrm{~nm}$. The steam band is stronger in the groundbased spectrum (Fig. 3), possibly due to the contribution of telluric absorption in the M99 spectrum. We also note that the STIS spectra are noisy in this region due to low quantum efficiency of the detector and the presence of a strong interference pattern at these red wavelengths. The PC3 index method yields a spectral type that is 2 subclasses earlier than the spectrum match, suggesting that this index method is not very reliable for the latest $\mathrm{L}$ dwarfs.

- DENIS-P J035726.9-441730 A (dM9): as shown in Fig. 4, the best match spectrum from M99 is that of DENIS-P 1431-1953 (dM9). In the ground-based spectrum the region from $740 \mathrm{~nm}$ to $770 \mathrm{~nm}$ is affected by telluric absorption. The PC3 index method also gives a subclass of dM9, an index in perfect agreement with the spectrum match.

- DENIS-P J035726.9-441730 B (dL1.5): as shown in Fig. 4, the best match spectrum from M99 is that of DENIS-P 1441-0945 (dL1). In the ground-based spectrum, the region from $740 \mathrm{~nm}$ to $770 \mathrm{~nm}$ is affected by telluric absorption so the mismatch between the STIS data and the M99 data over this wavelength coverage is not surprising. A similar discrepancy is seen in the spectra of all our late-M and early-L dwarfs. The PC3 index method gives a slightly later subclass of dL1.6. As a result, we average the two methods and round our adopted subclass to dL1.5.

- DENIS-P J100428.3-114648 A (dM9.5): both the PC3 index method and the best match spectrum (DENIS-P 1208+0149) give a spectral subclass of dM9.5 (Fig. 5).

- DENIS-P J100428.3-114648 B (dL0.5): the PC3 index method gives dM9.9. However, the STIS spectrum appears to yield an intermediate value, one between a dL0 (DENIS-P 0909-0658) and a dL1 (DENIS-P 1441-0945) as illustrated in Fig. 5. Therefore, we interpolate between a dL0 and a dL1 to obtain a subclass of dL0.5.

- DENIS-P J122821.6-241541 A (dL4): the PC3 index method gives dL3.9, and the M99 spectrum of LHS 102 B (dL4) is the best spectral match (Fig. 6, top). The Li I resonance doublet is not detected even though it has been clearly seen from the ground (Martín et al. 1997; Tinney et al. 1997). This nondetection is due to the low signal-to-noise ratio and to low resolution of our STIS data.

- DENIS-P J122821.6-241541 B (dL4.5): the PC3 index method gives dL4.2, and the M99 spectrum of DENIS-P J1228-2415 (bdL4.5) is the best spectral match (Fig. 6, bottom). The Li I resonance doublet is not detected because of the low signal-to-noise ratio of our data at $670.8 \mathrm{~nm}$.

- DENIS-P J144137.3-094559 A (dL1): the PC3 index method gives dL1.3, and the best M99 match is DENIS-P J1441-0945 itself (Fig. 7). We note that DENIS-P J144137.3-094559 is a common proper motion companion to the star G124-62 (dM4.5, Seifahrt et al. 2005), and both are members of the Hyades supercluster.

- GJ $569 \mathrm{Ba}$ (dM9): the PC3 index method gives a subtype of dM9.2, and the best spectral match is DENIS-P 1431-1953 (dM9). Thus, good agreement between the two methods is seen.

- GJ 569 Bb (dM9): the PC3 index method gives a subtype of dM8.6, but the best match is also DENIS-P 1431-1953 (Fig. 8). This example underlines the benefits of using several criteria to determine accurate spectral subclasses among ultracool dwarfs. If only the PC3 index method is used, then we would have assigned a spectral class to GJ $569 \mathrm{Bb}$ that is slightly earlier than that assigned to GJ $569 \mathrm{Ba}$, yielding results not consistent with the properties of this binary.

- 2MASSW J0746425+200032 A (dL0): the PC3 index method gives a subclass of dM9.5 but the best match is DENIS-P 0909-0658 (dL0) as shown in Bouy et al.

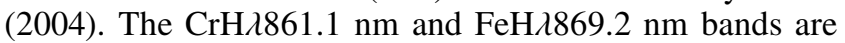
stronger than those seen in $\mathrm{M}$ dwarfs. However, the $\mathrm{CrH}$ and $\mathrm{FeH}$ bands are not stronger than the nearby $\mathrm{TiO}$ band at $843.2 \mathrm{~nm}$.

- 2MASSW J0746425+200032 B (dL1.5): the PC3 index method gives a subclass of dM9.6, but the best match is intermediate between DENIS-P 1441-0945 (dL1) and Kelu 1 (dL2), as shown in Bouy et al. (2004). As compared with the bands in the $\mathrm{A}$ component, the $\mathrm{TiO}$ and $\mathrm{VO}$ bands are weaker, and the $\mathrm{CrH}$ and $\mathrm{FeH}$ bands are stronger.

- 2MASSW J0920122+351742 (dL5): the PC3 index method gives a subclass of dL5.2, and the best match is DENIS-P J0205-1159 (dL5) as shown in Fig. 9. Once again, good agreement is seen between the two methods. 
- 2MASSW J1146344+223052 A (dL2): the PC3 index method gives a subclass of dL1.4, and the best spectral match is Kelu 1 (bdL2) as shown in Fig. 10. The K I resonance lines become so broad that the doublet is blended. The lines are clearly broader than the lines for the $\mathrm{dL} 1$. The $\mathrm{CrH}$ and $\mathrm{FeH}$ bands are stronger and the $\mathrm{TiO}$ bands are weaker than those bands for the dL1.

- 2MASSW J1146344+223052 B (dL2): the PC3 index method gives a subclass of $\mathrm{dL} 1.7$, and the best spectral match is Kelu 1 (bdL2) as shown in Fig. 10. Bouy et al. (2003) report a magnitude difference of 0.75 mag. in the $F 814 \mathrm{~W}$ filter, a value that is confirmed by new ACS images (H. Bouy 2006, private communication). The similar spectral types but different brightnesses of these two dwarfs suggest that 2MASSW J1146344+223052 A could be an unresolved binary with nearly equal components. Thus, 2MASSW J1146344+223052 may be a triple system. Very low-mass triple systems may not be rare as indicated by the recent observations of GJ 900 (Martín 2003) and DENIS-P J020529.0-115925 (Bouy et al. 2005). Confirmation of the suspected unresolved binarity of 2MASSW J1146344+223052 A requires high angular resolution observations or radial velocity monitoring.

- 2MASSW J1311391+803222 A, B (dM7.5, dM8). As shown in Fig. 11, the STIS spectra of both components are nearly identical. The spectral type inferred from the PC3 index method is dM7.3 for A and dM7.6 for B. The best spectral match for the A component is LHS2243 (dM7.5). The best spectral match for the B component is VB10 (dM8). The two lines of the $\mathrm{K}$ I resonance doublet can be distinguished with our spectral resolution. The $766.5 \mathrm{~nm}$ line is stronger than the $769.9 \mathrm{~nm}$ line. The Cs I is too weak to be seen in our spectra. The $\mathrm{TiO}$ and $\mathrm{VO}$ bands are conspicuous, and the small 0.5 subclass difference is best seen in the VO band at $740 \mathrm{~nm}$.

- 2MASSW J1426316+155701 A (dM8) and B (dL1.5): the spectrum of component A is very similar to that of VB10, a result that agrees with the $\mathrm{dM} 8$ subclass obtained from the PC3 measurement. The spectrum of component B is intermediate between that of DENIS-P J1441-0945 (dL1) and Kelu 1 (bdL2) as illustrated in Fig. 12.

\section{Discussion}

\subsection{Chromospheric activity and binarity}

$\mathrm{H}_{\alpha}$ emission is an indicator of chromospheric activity due to nonthermal heating. This heating results most likely from twisted magnetic field lines (that are frozen-in to the plasma) that break, releasing energy that accelerates charged (i.e., nonthermal) particles from the corona into the more-dense chromosphere, producing Bremsstrahlung radiation there. We have detected $\mathrm{H}_{\alpha}$ emission in only two of our objects. We give the equivalent width measurements or upper limits in Table 3 . We did not see any flares, which usually display a variety of strong emission lines in late-M and L dwarfs (Liebert et al. 1999; Martín 1999; Martín \& Ardila 2001).

In Fig. 13, we display a zoom of the $\mathrm{H}_{\alpha}$ region for most of our targets. Our three coolest L dwarfs (dL5-dL6) are missing from the plot because they do not have enough continuum for equivalent width determination at these wavelengths. We compare the distribution of $\mathrm{H}_{\alpha}$ emission equivalent width, with respect to spectral subclass in our sample, with the measurements obtained by Gizis et al. (2000) for field dwarfs. We perform

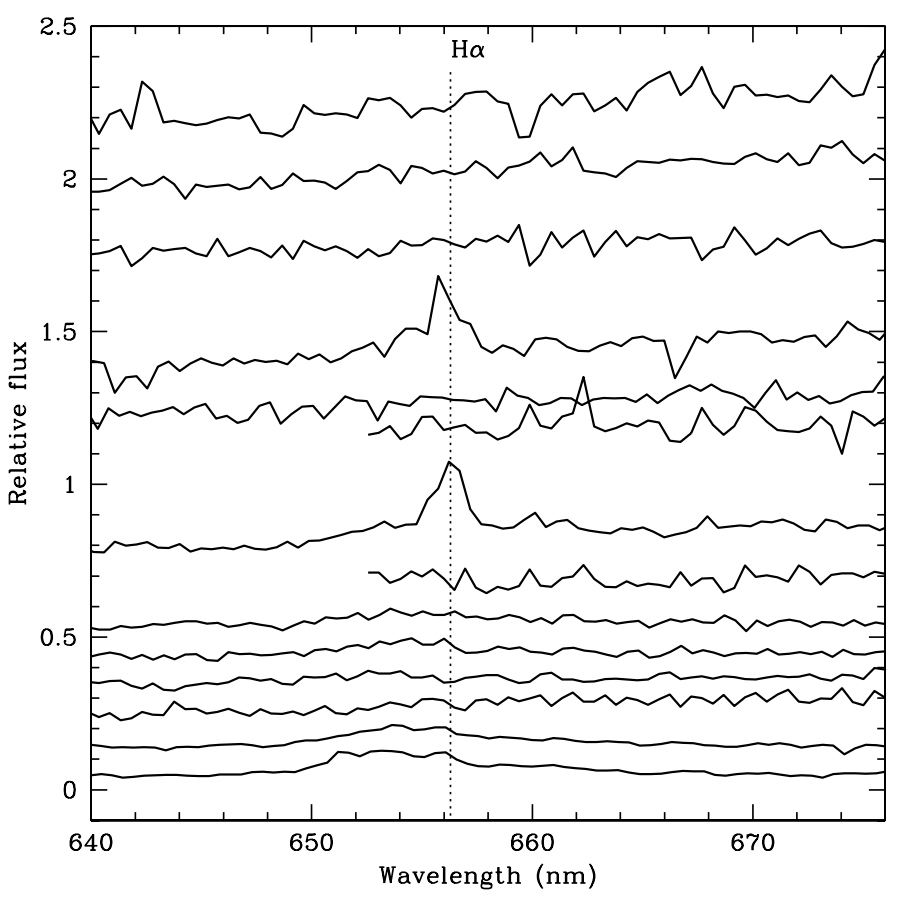

Fig. 13. A zoom of the STIS spectral region around the $\mathrm{H}_{\alpha}$ emission line. In order of increasingly late spectral subclass from bottom to top: 2MASS $\mathrm{J} 1311+8032 \mathrm{~A}$ and $\mathrm{B}$, 2MASS J1146+2230 A, DENIS-P 0357-4417 A, Gl 569 Ba and Bb, DENIS J1004-1146 A, 2MASS J0746+2000 A, DENIS J1004-1146 B, DENIS-P 1441-0945 A, 2MASS J0746+2000 B, 2MASS J1146+2230 B, DENIS J1228-1547 A and B.

a Kolmogorov-Smirnov test to check whether the two distributions of $\mathrm{H}_{\alpha}$ emission equivalent width values are statistically different or similar. We bin the data in steps of 0.5 subclasses, yielding six spectral bins in the range dM7-dM9.5. We find that the two distributions of spectral types are $90 \%$ similar, but their associated distributions of $\mathrm{H}_{\alpha}$ equivalent widths are different with high level of confidence (99.9\%). Our sample of resolved binary components tends to show lower $\mathrm{H}_{\alpha}$ activity than single field dwarfs in the spectral range dM7-dM9.5. The level of activity is unclear for $\mathrm{L}$ dwarfs because the datapoints are too few.

Our results indicate an anti-correlation between chromospheric activity and resolved binarity for the latest dMs. This anti-correlation may be due to different angular momentum histories in the binary components, although unlikely as the connection between activity and rotation for these late dwarfs is not good (Mohanty \& Basri 2003). On the other hand, chromospheric activity has been suggested to be enhanced in some latetype dwarfs after impact of asteroids or comets (e.g. AB Dor, Gómez de Castro 2002). A "planetesimal-impact" hypothesis to explain flares in cool stars has also been discussed in the literature (Hertzsprung 1924; Andrews 1991). Our results may lend support to this hypothesis as the binaries in our sample may have less debris material due to disk clearing by the components of the binary system. In single, very-late $\mathrm{dMs}$, debris material may last for longer periods of time than for solar-type stars because of the reduced effect of disk dissipation processes such as the Poynting-Robertson drag.

\subsection{Alkali lines}

We search for the Li I resonance line in our spectra. This line is a useful diagnostic of the age and mass of ultracool dwarfs 


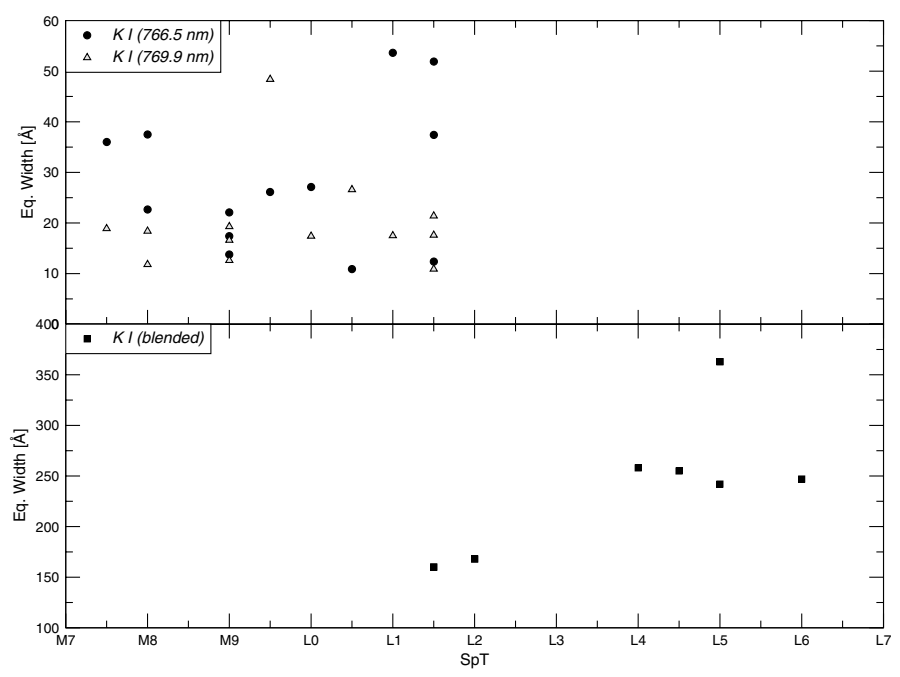

Fig. 14. Equivalent widths of the $\mathrm{K}$ I resonance doublet at 766.5 and $769.9 \mathrm{~nm}$ versus spectral type for program ultracool dwarfs.

(Magazzù et al. 1993; Martín et al. 1994). This line has been previously detected in the unresolved spectra of one of our program binaries with equivalent widths in the range 2-4 (DENIS-P J122821.6-241541 A, B; Martín et al. 1997; Tinney et al. 1997). However, we could not detect the line in our spectrum of DENIS-P J122821.6-241541 A. We conclude that the resolution and limited signal-to-noise ratio of our STIS spectra do not allow us to detect $\mathrm{Li}$ I resonance lines with equivalent widths smaller than about $5 \AA$, implying that we cannot set useful constraints on the lithium abundance in our program dwarfs.

We measure the $\mathrm{K}$ I resonance doublet at 766.5 and $769.9 \mathrm{~nm}$. For the dwarfs where we could distinguish the two lines, we used the Gaussian deblending option in the IRAF package splot. The equivalent widths obtained are given in Table 3 and their dependence with spectral class is illustrated in Fig. 14. At subclass dL2 and later, the doublet becomes extremely broad and the two lines are blended (Martín et al. 1997, 1999b; Burrows \& Sharp 1999; Kirkpatrick et al. 1999). For those dLs, we provide the equivalent width of the blended pair. The Na I subordinate doublet at 818.4 and $819.5 \mathrm{~nm}$ could not be measured because of a bad column in the array. The Cs I at 852.1 and $894.3 \mathrm{~nm}$ are strong enough in the coolest objects to measure the equivalent width (Table 3 ).

The scatter in the equivalent widths of the $\mathrm{K}$ I resonance doublet as a function of spectral class, as seen in Fig. 14, is likely due to the its well-known sensitivity to surface gravity (M99). For example, we note that GJ $569 \mathrm{Ba}$ and $\mathrm{Bb}$ (dM9) have weaker $\mathrm{K}$ I equivalent width than 2MASSW J1426316+155701 A (dM8) and DENIS-P J100428.3-114648 A (dM9.5). Weaker $\mathrm{K} I$ is an indication of lower surface gravity, and consequently a younger age and lower mass for a given spectral subclass. The age of GJ 569 B has been estimated to be young ( 300 Myr) by Zapatero Osorio et al. (2004) using evolutionary tracks. Therefore, the ages of DENIS-P J100428.3-114648 and 2MASSW J1426316+155701 are likely to be older than that of GJ 569 B. With more observations and detailed modelling, the $\mathrm{K}$ I resonance doublet may yield a useful age calibration for very low-mass stars and brown dwarfs.

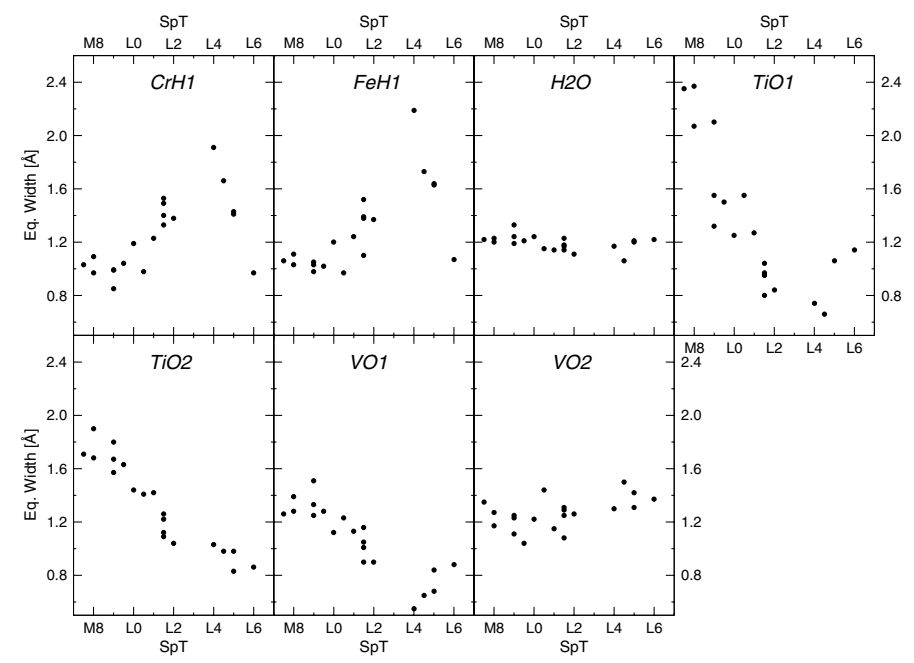

Fig. 15. Molecular absorption indices versus spectral type for program ultracool dwarfs.

\subsection{Molecular bands}

The main molecular bands in our spectra are labeled in Fig. 2. We measure their strengths using the indices defined in M99, and we give the values in Table 4. As already discussed in Kirkpatrick et al. (1999) and M99, the TiO bands diminish in strength from the late-M to the L dwarfs, and they eventually become undetectable in mid-L dwarfs. The bands dependence on spectral type is shown in Fig. 15. This disappearing effect is best explained by the settling of $\mathrm{Ti}$ onto dust grains, such as $\mathrm{CaTiO}_{3}$, at condensation temperatures below $2500 \mathrm{~K}$ (Allard et al. 2001). The VO molecule behaves in a similar way to the TiO but the disappearing effect occurs at a slightly cooler temperature (Fig. 15). The chromium and iron hydrides become prominent in mid-L dwarfs, and they tend to fade away in the late-L dwarfs (Fig. 15).

The values of the two TiO indices are lower in GJ $569 \mathrm{Ba}$ than in our dM7.5-dM8 targets, but the VO indices are more similar. This result may be another spectroscopic manifestation of low surface gravity. The weakening of TiO bands may be shifted to later spectral subclass in low gravity objects because of less efficient dust formation. Martín et al. (1996) noted that dM7-dM9 members of the young Pleiades cluster have stronger $\mathrm{TiO}$ bands than their older counterparts in the field.

\subsection{Comparison of spectral subclasses}

In Table 5, we show a comparison of the spectral subclasses adopted by us in the M99 system with those reported in the literature. The spectral types given in the literature correspond to the unresolved systems, and thus the spectral types are dominated by the primary. The table shows a fairly good agreement. No discrepancies larger than 2 subclasses are noted. The nearinfrared spectral types from Geballe et al. (2002) for two of our binaries also agree within 2 subclasses. The near-infrared spectral types of Gl $569 \mathrm{Ba}$ and b reported by Lane et al. (2001) are consistent with our optical spectral types within the uncertainties (half a subclass).

\subsection{Absolute magnitude versus spectral type relationships}

Among our sample, only five binaries have known parallaxes. Their properties are summarized in Table 6. In Fig. 16, we plot 
Table 6. Spectral types and photometric magnitudes for binaries with known parallaxes.

\begin{tabular}{lcccccc}
\hline \hline $\begin{array}{l}\text { Object } \\
\text { (abridged name) }\end{array}$ & $\begin{array}{c}\text { Sp.T. } \\
\text { (adopted) }\end{array}$ & $m-M$ & $M_{F 814 W}$ & $M_{J}$ & $M_{K \mathrm{~s}}$ & Refs. \\
\hline DENIS J0205-1159 A & dL5 & $1.48 \pm 0.06$ & $17.10 \pm 0.12$ & & $12.3 \pm 0.1$ & Bo03, Ko99 \\
DENIS J0205-1159 B & dL6 & & $18.18 \pm 0.12$ & & $12.3 \pm 0.1$ & Bo05, Ko99 \\
DENIS J1228-2415 A & dL4 & $1.53 \pm 0.01$ & $16.62 \pm 0.04$ & $13.56^{1} \pm 0.08$ & $12.0 \pm 0.1$ & Br04, Ko99, MBB99, V04 \\
DENIS J1228-2415 B & dL4.5 & & $16.71 \pm 0.04$ & $13.76^{1} \pm 0.08$ & $12.0 \pm 0.1$ & Ko99, MBB99 \\
GJ 569 Ba & dM9 & $-0.04 \pm 0.02$ & $12.54 \pm 0.16$ & $11.18 \pm 0.08$ & $10.06 \pm 0.09$ & L01 \\
GJ 569 Bb & dM9 & & $13.24 \pm 0.16$ & $11.69 \pm 0.08$ & $10.47 \pm 0.09$ & Bo04 \\
2MASS J0746+2000A & dL0 & $0.43 \pm 0.01$ & $14.98 \pm 0.16$ & $11.76 \pm 0.08$ & $10.60 \pm 0.03$ & Bo04 \\
2MASS J0746+2000B & dL1.5 & & $15.98 \pm 0.21$ & $12.36 \pm 0.22$ & $11.12 \pm 0.05$ & Ko99, Bo03, V04 \\
2MASS J1146+2230 A & dL2 & $2.83 \pm 0.05$ & $15.34 \pm 0.17$ & & $10.4 \pm 0.1$ & Bo03, Ko99 \\
2MASS J1146+2230 B & dL2 & & $16.09 \pm 0.22$ & & $10.6 \pm 0.1$ & \\
\hline
\end{tabular}

References: Br04 = Brandner et al. (2004), Bo03 = Bouy et al. (2003), Bo04 = Bouy et al. (2004), Bo05 = Bouy et al. (2005), G02 = Geballe et al. (2002), K99 = Kirkpatrick et al. (1999), Ko99 = Koerner et al. (1999), L01 = Lane et al. (2001), MBB99 = Martín et al. (1999a), V04 = Vrba et al. (2004). ${ }^{1} M_{J}$ deduced from F110M data from Martín et al. (2000). ${ }^{2}$ DENIS-P J0205-1159 B is itself likely double (Bouy et al. 2005). Our STIS spectrum and the $K$ s magnitude of Koerner et al. (1999) include the sum of the two components.
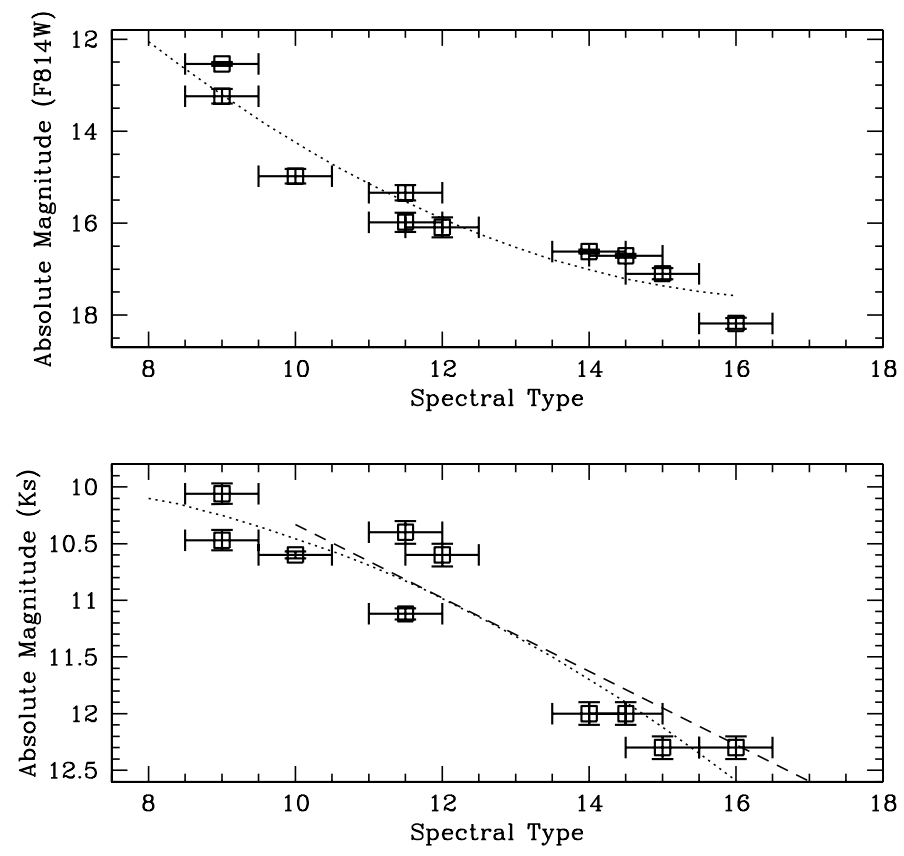

Fig. 16. Absolute magnitude in the HST F814W filter (top panel) and $K$-band (bottom panel) versus spectral type for program ultracool dwarfs. A second order polynomial fit to the data is shown with a dotted line. The dashed straight line represents the linear relatioship reported by Vrba et al. (2004).

the spectral types of their resolved components versus the absolute magnitudes in the photometric bands $F 814 \mathrm{~W}$ and $K_{\mathrm{s}}$. As expected, later type objects are cooler and have fainter magnitudes. Using second order polynomials, we find that the following equations provide good fits to our data in the spectral class range dM9-dL6:

$M_{F 814 W}=-2.2167+2.3284 \times S p T-0.0682 \times S p T^{2}$

$M_{K \mathrm{~s}}=10.502-0.2311 \times S p T+0.0226 \times S p T^{2}$

where we used the numerical code of $S p T=9$ for M9 through $S p T=16$ for L6. These fits are shown as dotted lines in Fig. 16. The scatter $(1 \sigma)$ in relations 1 and 2, over the sample of objects that we fit these relations to, is $0.43 \mathrm{mag}$ and $0.27 \mathrm{mag}$, respectively. The good correlation between our adopted spectral types and the absolute magnitudes indicates that the late-M and L dwarf spectral classification scale depends primarily on effective temperature. The observed scatter around these relations is likely due to the added contributions of unresolved binarity (higher order multiple systems), observational errors, gravity effects, and metallicity differences in the sample. Our results are consistent with those reported by other authors such as Kirkpatrick et al. (2000), Dahn et al. (2002) and Vrba et al. (2004) within the observational error bars. A comparison of our $S p T-M_{K_{\mathrm{s}}}$ relationship with that of Vrba et al. (2004) is shown in Fig. 16.

\section{Summary}

We present low-resolution $(R=470)$, optical spectroscopy of 20 ultracool dwarfs in resolved binary systems. Eighteen targets are members of nine resolved binaries with angular separations in the range 0.'37-0.'098.

We derive spectral types for our targets using the PC3 index method and by direct comparison with the M99 ground-based spectra. The second method is preferred over the first one in the few cases that show discrepancies larger than 1 spectral subclass because the second method includes more spectral information than the first one. We report that the $\mathrm{H}_{\alpha}$ emission in our targets is statistically weaker than that found in field dwarfs for the range of spectral class dM7-dM9.5. We did not detect any flares. We did not detect the Li I resonance doublet in our targets because of the poor quality and low resolution of our STIS spectra. We provide pseudo-equivalent widths of the $\mathrm{Cs} \mathrm{I}$ and $\mathrm{K}$ I resonance doublets for those targets that have lines that could be measured. These lines tend to increase in breath and strength toward later spectral types, as already reported by Kirkpatrick et al. (1999) and M99. However, significant dispersion is seen, a result that may be due to gravity effects. This doublet may serve as a useful age indicator for field ultracool dwarfs.

For a subset of 10 targets in 5 binaries with known trigonometric parallaxes, we show good correlation between our spectral types and the absolute magnitudes of the targets in the $F 814 \mathrm{~W}$ and $K_{\mathrm{s}}$ bands. We provide second order polynomial fits to the data. These fits could then be used to derive spectrophotometric parallaxes of late-M and L field dwarfs.

After publication of this paper, we plan to make the spectra available online via the IAC catalog of ultracool dwarfs. The address of this catalog is: http://www.iac.es/ galeria/ege/catalogo_espectral/. The description of the catalog can be found in Martín et al. (2005). 
Acknowledgements. We thank Jay Anderson and the staff at STScI for help with the STIS observations. This work is based on observations collected with the NASA/ESA Hubble Space Telescope operated at the Space Telescope Science Institute (STSci), programs G09157, GO9451, and GO9499. The STSci is operated by the Association of Universities for Research in Astronomy, Inc., under NASA contract NAS 5-26555. Research presented herein was partially funded by NSF research grant AST 02-05862, by the Deutsches Zentrum für Luft- und Raumfahrt (DLR), Förderkennzeichen 50 OR 0401, and by Spanish MEC AYA2005-06453.

\section{References}

Abt, H. A., \& Levy, S. G. 1973, AJ, 78, 1093

Andrews, A. D. 1991, A\&A, 245, 219

Billères, M., Delfosse, X., Beuzit, J.-L., et al. 2005, A\&A, 440, L55

Bouy, H., Brandner, W., Martín, E. L., et al. 2003, AJ, 126, 1526

Bouy, H., Duchêne, G., Köhler, R., et al. 2004, A\&A, 423, 341

Bouy, H., Martín, E. L., Brandner, W., \& Bouvier, J. 2005, ApJ, 129, 511

Brandner, W., Martín, E. L., Bouy, H., et al. 2004, A\&A, 428, 205

Burgasser, A. J., \& McElwain, M. W. 2006, AJ, 131, 1007

Burgasser, A. J., Kirkpatrick, J. D., Reid, I. N., et al. 2003, ApJ, 586, 512

Burrows, A., \& Sharp, C. 1999, ApJ, 412, 843

Chang, K. 1972, AJ, 77, 759

Chauvin, G., Lagrange, A.-M., Dumas, C., et al. 2004, A\&A, 425, L29

Close, L. M., Siegler, N., Freed, M., \& Biller, B. 2003, ApJ, 587, 407

Couteau, P. 1957, Journal des Observateurs, 40, 126

Dahn, C. C., Harris, H. C., Vrba, F. J., et al. 2002, AJ, 124, 1170

Delfosse, X., Forveille, T., Ségransan, D., et al. 2000, A\&A, 364, 217

Forrest, W. J., Shure, M., \& Skrutskie, M. F. 1988, ApJ, 567, L59

Geballe, T. R., Knapp, G. R., Leggett, S. K., et al. 2002, ApJ, 564, 466

Gizis, J. E., Monet, D. G., Reid, I. N., et al. 2000, AJ, 120, 1085

Gizis, J. E., Reid, I. N., Knapp, G. R., et al. 2003, AJ, 125, 3302

Golimowski, D. A., Henry, T. J., Krist, J. E., et al. 2004, AJ, 128, 1733

Gómez de Castro, A. I. 2002, MNRAS, 332, 409

Goto, M., Kobayashi, N., Terada, H., et al. 2002, ApJ, 567, L59

Hertzsprung, E. 1924, Bull. Astron. Netherlands, 2, 87

Kirkpatrick, J. D., Reid, I. N., Liebert, J., et al. 1999, AJ, 519, 802

Kirkpatrick, J. D., Reid, I. N., Liebert, J., et al. 2000, AJ, 120, 447

Koerner, D. W., Kirkpatrick, J. D., McElwain, M. W., \& Bonaventura, N. R. 1999, ApJ, 526, L25
Kraus, A. L., White, R. J., \& Hillebrand, L. A. 2005, ApJ, 633, 452

Kroupa, P., Bouvier, J., Duchene, G., \& Moraux, E. 2003, MNRAS, 346, 354

Lane, B. F., Zapatero Osorio, M. R., Britton, M. C., Martín, E. L., \& Kulkarni, S. R. 2001, ApJ, 560, 390

Leggett, S. K., Golimowski, D. A., Fan, X., et al. 2002, ApJ, 564, 452

Liebert, J., Kirkpatrick, J. D., Reid, I. N., \& Fisher, M. D. 1999, ApJ, 519, 345

Luhman, K. 2004, ApJ, 614, 398

Luhman, K. 2005, ApJ, 633, L41

Magazzù, A., Martín, E. L., \& Rebolo, R. 1993, ApJ, 404, L17

Martín, E. L. 1999, MNRAS, 302, 59

Martín, E. L. 2003, AJ, 126, 918

Martín, E. L., \& Ardila, D. R. 2001, AJ, 121, 2758

Martín, E. L., Rebolo, R., \& Zapatero Osorio, M. R. 1996, ApJ, 469, 706

Martín, E. L., Basri, G., Delfosse, X., \& Forveille, T. 1997, A\&A, 327, L29

Martín, E. L., Basri, G., Brandner, W., et al. 1998, ApJ, 509, L113

Martín, E. L., Brandner, W., \& Basri, G. 1999a, Science, 283, 1718

Martín, E. L., Delfosse, X., Basri, G., et al. 1999b, AJ, 118, 2466

Martín, E. L., Koresko, C. D., Kulkarni, S. R., Lane, B. F., \& Wizinowich, P. L. 2000a, ApJ, 529, L37

Martín, E. L., Brandner, W., Bouvier, J., et al. 2000b, ApJ, 543, 299

Martín, E. L., Cabrera, J., \& Cenizo, E. 2005, AN, 326, 1026

McCaughrean, M. J., Close, L. M., Scholz, R.-D., et al. 2004, A\&A, 413, 1029

Mohanty, S., \& Basri, G. 2003, ApJ, 583, 451

Phan Bao, N., Martín, E. L., Reylé, C., Forveille, T., \& Lim, J. 2005, A\&A, 439, L19

Potter, D., Martín, E. L., Cushing, M. C., et al. 2002, ApJ, 567, L133

Reid, I. N., Gizis, J. E., Kirkpatrick, J. D., \& Koerner, D. W. 2001, AJ, 121, 489

Schweitzer, A., Gizis, J. E., Hauschildt, P. H., Allard, F., \& Reid, I. N. 2001, ApJ, 555, 368

Seifahrt, A., Guenther, E., \& Neuhauser, R. 2005, A\&A, 440, 967

Siegler, N., Close, L., Cruz, K. L., Martín, E. L., \& Reid, I. N. 2005, 621, 1023

Simon, M., Bender, C., \& Prato, L. 2006, ApJ, in press [arXiv: astro-ph/0603165]

Stassun, K., Mathieu, R. D., \& Valenti, J. 2006, Nature, 440, 311

Umbreit, S., Burkert, A., Henning, T., Mikkola, S., \& Spurzem, R. 2005, ApJ, 623,940

Tinney, C. G., Delfosse, X., \& Forveille, T. 1997, ApJ, 490, L95

van de Kamp, P. 1938, AJ, 47, 168

Vrba, F. J., Henden, A. A., Luginbuhl, C. B., et al. 2004, AJ, 127, 2948

Zapatero Osorio, M. R., Lane, B. F., Pavlenko, Ya., et al. 2004, ApJ, 615, 958 
E. L. Martín et al.: Resolved HST spectroscopy of ultracool binaries, Online Material $p$ I

\section{Online Material}


Table 1. STIS observing log.

\begin{tabular}{|c|c|c|c|c|c|}
\hline Object & $\begin{array}{c}\text { Exp. } \\
\text { Time [s] }\end{array}$ & $\begin{array}{c}\text { Date Obs. } \\
\text { DD/MM/YYYY }\end{array}$ & Program & $\begin{array}{l}\text { Ref. object } \\
\text { discovery }\end{array}$ & $\begin{array}{l}\text { Ref. binarity } \\
\text { discovery }\end{array}$ \\
\hline DENIS-P J144137.3-094559 A & 4695 & $29 / 03 / 2002$ & GO9157 & Martín et al. (1999b) & Bouy et al. (2003) \\
\hline DENIS-P J122821.6-241541 A,B & 4693 & $25 / 04 / 2002$ & GO9157 & Delfosse et al. (1998) & Martín et al. (1999a) \\
\hline GJ $569 \mathrm{Ba}, \mathrm{Bb}$ & 3873 & $26 / 06 / 2002$ & GO9499 & Forrest et al. (1988) & Martín et al. (2000a) \\
\hline DENIS-P J020529.0-115925 A,B & 4695 & $25 / 09 / 2002$ & GO9157 & Delfosse et al. (1997) & Koerner et al. (1999) \\
\hline DENIS-P J035726.9-441730 A,B & 4895 & 08/01/2003 & GO9451 & Bouy et al. (2003) & Bouy et al. (2003) \\
\hline 2MASSW J1146344+223052 A,B & 4702 & $10 / 02 / 2003$ & GO9157 & Kirkpatrick et al. (1999) & Koerner et al. (1999) \\
\hline 2MASSW J1311391+803222 A,B & 4183 & $27 / 02 / 2003$ & GO9451 & Gizis et al. (2000) & Close et al. (2003) \\
\hline 2MASSW J0920122+351742 & 4774 & $10 / 03 / 2003$ & GO9451 & Kirkpatrick et al. (2000) & Bouy et al. (2003) \\
\hline DENIS-P J100428.3-114648 A,B & 4324 & $14 / 03 / 2003$ & GO9451 & Bouy et al. (2003) & Bouy et al. (2003) \\
\hline 2MASSW J1426316+155701 A,B & 1980 & 28/04/2003 & GO9451 & Gizis et al. (2000) & Close et al. (2003) \\
\hline 2MASSW J0746425+200032 A,B & 1980 & $09 / 01 / 2004$ & GO9451 & Kirkpatrick et al. (2000) & Reid et al. (2001) \\
\hline
\end{tabular}

Table 2. PC3 index and spectral subclasses.

\begin{tabular}{|c|c|c|c|c|}
\hline $\begin{array}{l}\text { Name of } \\
\text { object }\end{array}$ & $\overline{\mathrm{PC} 3}$ & $\begin{array}{l}\text { SpT } \\
\text { (PC3) }\end{array}$ & $\begin{array}{c}\mathrm{SpT} \\
\text { (best match) }\end{array}$ & $\begin{array}{c}\text { SpT } \\
\text { (adopted) }\end{array}$ \\
\hline DENIS-P J020529.0-115925 A & 6.21 & $\mathrm{dL} 4.1$ & $\mathrm{dL} 5$ & dL5 \\
\hline DENIS-P J020529.0-115925 B & 5.48 & dL3.6 & dL6 & dL6 \\
\hline DENIS-P J035726.9-441730 A & 2.15 & dM9.1 & dM9 & dM9 \\
\hline DENIS-P J035726.9-441730 B & 2.94 & $\mathrm{dL} 1.6$ & $\mathrm{dL} 1$ & $\mathrm{dL} 1.5$ \\
\hline DENIS-P J100428.3-114648 A & 2.33 & dM9.6 & dM9-dM9.5 & dM9.5 \\
\hline DENIS-P J100428.3-114648 B & 2.49 & dM9.9 & $\mathrm{dL} 0-\mathrm{dL} 1$ & dL0.5 \\
\hline DENIS-P J122821.6-241541 A & 5.92 & dL3.9 & dL4 & dL4 \\
\hline DENIS-P J122821.6-241541 B & 6.39 & $\mathrm{dL} 4.2$ & dL4.5 & dL4.5 \\
\hline DENIS-P J144137.3-094559 A & 2.56 & $\mathrm{dL} 1.3$ & dL1 & dL1 \\
\hline GJ $569 \mathrm{Ba}$ & 2.17 & dM9.2 & dM9 & dM9 \\
\hline GJ $569 \mathrm{Bb}$ & 1.99 & dM8.6 & dM9 & dM9 \\
\hline 2MASSW J0746425+200032 A & 2.29 & dM9.5 & dL0 & $\mathrm{dL} 0$ \\
\hline 2MASSW J0746425+200032 B & 2.34 & dM9.6 & dL1.5 & $\mathrm{dL} 1.5$ \\
\hline 2MASSW J0920122+351742 & 8.58 & dL5.2 & dL5 & dL5 \\
\hline 2MASSW J1146344+223052 A & 2.69 & $\mathrm{dL} 1.4$ & $\mathrm{dL} 2$ & $\mathrm{dL} 2$ \\
\hline 2MASSW J1146344+223052 B & 3.07 & $\mathrm{dL} 1.7$ & $\mathrm{dL} 2$ & $\mathrm{dL} 2$ \\
\hline 2MASSW J1311391+803222 A & 1.68 & dM7.3 & dM7.5 & dM7.5 \\
\hline 2MASSW J1311391+803222 B & 1.75 & dM7.6 & dM8 & $\mathrm{dM} 8$ \\
\hline 2MASSW J1426316+155701 A & 1.78 & dM7.7 & dM8 & dM8 \\
\hline 2MASSW J1426316+155701 B & 2.75 & $\mathrm{dL} 1.4$ & dL1-dL2 & $\mathrm{dL} 1.5$ \\
\hline
\end{tabular}


E. L. Martín et al.: Resolved HST spectroscopy of ultracool binaries, Online Material p 3

Table 3. Atomic line data.

\begin{tabular}{lccccc}
\hline \hline Name of & $\mathrm{H}_{\alpha}$ & $\mathrm{K} \mathrm{I}$ & $\mathrm{K} \mathrm{I}$ & $\mathrm{Cs} \mathrm{I}$ & $\mathrm{Cs} \mathrm{I}$ \\
object & 656.3 & 766.5 & 769.9 & 852.1 & 894.3 \\
\hline DENIS-P J020529.0-115925 A & $\ldots$ & $242^{1}$ & $\ldots$ & 4.2 & 3.9 \\
DENIS-P J020529.0-115925 B & $\ldots$ & $247^{1}$ & $\ldots$ & 7.5 & 5.1 \\
DENIS-P J035726.9-441730 A & $<-3$ & 17.4 & 12.6 & $\ldots$ & 2.9 \\
DENIS-P J035726.9-441730 B & $<-6$ & 12.4 & 10.9 & $\ldots$ & $\ldots$ \\
DENIS-P J100428.3-114648 A & $<-3$ & 26.1 & 48.4 & $\ldots$ & $\ldots$ \\
DENIS-P J100428.3-114648 B & $<-3$ & 10.9 & 26.6 & $\ldots$ & $\ldots$ \\
DENIS-P J122821.6-241541 A & $<-5$ & $258^{1}$ & $\ldots$ & $12.4:^{2}$ & 4.5 \\
DENIS-P J122821.6-241541 B & $<-5$ & $255^{1}$ & $\ldots$ & $6.6:^{2}$ & $2.2:^{2}$ \\
DENIS-P J144137.3-094559 A & $<-3$ & 53.6 & 17.5 & $4.3:^{2}$ & $\ldots$ \\
GJ 569 Ba & $<-3$ & 13.8 & 16.6 & $\ldots$ & $\ldots$ \\
GJ 569 Bb & $<-3$ & 22.1 & 19.3 & $\ldots$ & $\ldots$ \\
2MASSW J0746425+200032 A & -18.8 & 27.1 & 17.4 & $\ldots$ & $\ldots$ \\
2MASSW J0746425+200032 B & -19.1 & 37.4 & 17.6 & $\ldots$ & $\ldots$ \\
2MASSW J0920122+351742 & $\ldots$ & $363^{1}$ & $\ldots$ & $\ldots$ & 4.3 \\
2MASSW J1146344+223052 A & $<-6$ & $160^{1}$ & $\ldots$ & 3.5 & 2.4 \\
2MASSW J1146344+223052 B & $<-7$ & $168^{1}$ & $\ldots$ & 4.6 & $4.1:^{2}$ \\
2MASSW J1311391+803222 A & $<-3$ & 36.0 & 18.9 & $\ldots$ & $\ldots$ \\
2MASSW J1311391+803222 B & $<-3$ & 22.7 & 18.4 & $\ldots$ & $\ldots$ \\
2MASSW J1426316+155701 A & $<-3$ & 37.5 & 11.8 & $\ldots$ & $\ldots$ \\
2MASSW J1426316+155701 B & $<-3$ & 51.9 & 21.4 & $\ldots$ & $\ldots$ \\
\hline
\end{tabular}

Note. Equivalent width values are in given angströms and wavelegths are given in nanometers. 1- $\sigma$ uncertainties typically are $\sim 15 \%$ of the equivalent width. ${ }^{1}$ Corresponds to the blend of the 7665 and $7699 \AA$ doublet. Integration limits $734.0-787.0 \mathrm{~nm} ;{ }^{2}$ uncertain measurement due to blending or noise.

Table 4. Molecular band and pseudocontinuum slope data.

\begin{tabular}{lccccccc}
\hline \hline Object & CrH1 & FeH1 & $\mathrm{H}_{2} \mathrm{O}$ & $\mathrm{TiO} 1$ & $\mathrm{TiO} 2$ & $\mathrm{VO} 1$ & $\mathrm{VO} 2$ \\
\hline DENIS-P J020529.0-115925 A & 1.64 & 1.43 & 1.20 & 1.06 & 0.98 & 0.84 & 1.42 \\
DENIS-P J020529.0-115925 B & 1.07 & 0.97 & 1.22 & 1.14 & 0.86 & 0.88 & 1.37 \\
DENIS-P J035726.9-441730 A & 1.03 & 0.85 & 1.33 & 1.32 & 1.80 & 1.51 & 1.23 \\
DENIS-P J035726.9-441730 B & 1.10 & 1.33 & 1.23 & 0.97 & 1.09 & 1.05 & 1.29 \\
DENIS-P J100428.3-114648 A & 1.02 & 1.04 & 1.21 & 1.50 & 1.63 & 1.28 & 1.04 \\
DENIS-P J100428.3-114648 B & 0.97 & 0.98 & 1.15 & 1.55 & 1.41 & 1.23 & 1.44 \\
DENIS-P J122821.6-241541 A & 2.19 & 1.91 & 1.17 & 0.74 & 1.03 & 0.55 & 1.30 \\
DENIS-P J122821.6-241541 B & 1.73 & 1.66 & 1.06 & 0.66 & 0.98 & 0.65 & 1.50 \\
DENIS-P J144137.3-094559 A & 1.24 & 1.23 & 1.14 & 1.27 & 1.42 & 1.13 & 1.15 \\
GJ 569 Ba & 1.05 & 0.99 & 1.24 & 1.55 & 1.57 & 1.25 & 1.25 \\
GJ 569 Bb & 0.98 & 0.99 & 1.19 & 2.10 & 1.67 & 1.33 & 1.11 \\
2MASSW J0746425+200032 A & 1.20 & 1.19 & 1.24 & 1.25 & 1.44 & 1.12 & 1.22 \\
2MASSW J0746425+200032 B & 1.39 & 1.40 & 1.18 & 1.04 & 1.22 & 1.01 & 1.25 \\
2MASSW J0920122+351742 & 1.63 & 1.41 & 1.21 & 0.40 & 0.83 & 0.68 & 1.31 \\
2MASSW J1146344+223052 A & 1.52 & 1.53 & 1.17 & 0.80 & 1.12 & 0.90 & 1.31 \\
2MASSW J1146344+223052 B & 1.37 & 1.38 & 1.11 & 0.84 & 1.04 & 0.90 & 1.26 \\
2MASSW J1311391+803222 A & 1.06 & 1.03 & 1.22 & 2.35 & 1.71 & 1.26 & 1.35 \\
2MASSW J1311391+803222 B & 1.03 & 0.97 & 1.20 & 2.37 & 1.68 & 1.39 & 1.27 \\
2MASSW J1426316+155701 A & 1.11 & 1.09 & 1.23 & 2.07 & 1.90 & 1.28 & 1.17 \\
2MASSW J1426316+155701 B & 1.38 & 1.49 & 1.14 & 0.95 & 1.26 & 1.16 & 1.08 \\
\hline
\end{tabular}

Note. Integration limits for these spectral indices are given in M99. 
E. L. Martín et al.: Resolved HST spectroscopy of ultracool binaries, Online Material p 4

Table 5. Comparison of optical spectral types.

\begin{tabular}{lccc}
\hline \hline Object & This work & M99 & K99, 00 \\
\hline DENIS-P J020529.0-115925 A & dL5 & dL5 & L7 \\
DENIS-P J020529.0-115925 B & dL6 & & \\
DENIS-P J122821.6-241541 A & dL4 & bdL4.5 & L5 \\
DENIS-P J122821.6-241541 B & dL4.5 & & \\
DENIS-P J144137.3-094559 A & dL1 & dL1 & \\
GJ 569 Ba & dM9 & dM8.5 & \\
GJ 569 Bb & dM9 & & \\
2MASSW J0746425+200032 A & dL0 & & dL0.5 \\
2MASSW J0746425+200032 B & dL1.5 & & \\
2MASSW J0920122+351742 A & dL5 & & L3 \\
2MASSW J1146344+223052 A & dL2 & & \\
2MASSW J1146344+223052 B & dL2 & & \\
2MASSW J1311391+803222 A & dM7.5 & & \\
2MASSW J1311391+803222 B & dM8 & & \\
2MASSW J1426316+155701 A & dM8 & & \\
2MASSW J1426316+155701 B & dL1.5 & \\
\hline
\end{tabular}

References: K99: Kirkpatrick et al. (1999); K00: Kirkpatrick et al. (2000); M99: Martín et al. (1999b). 
E. L. Martín et al.: Resolved HST spectroscopy of ultracool binaries, Online Material p 5

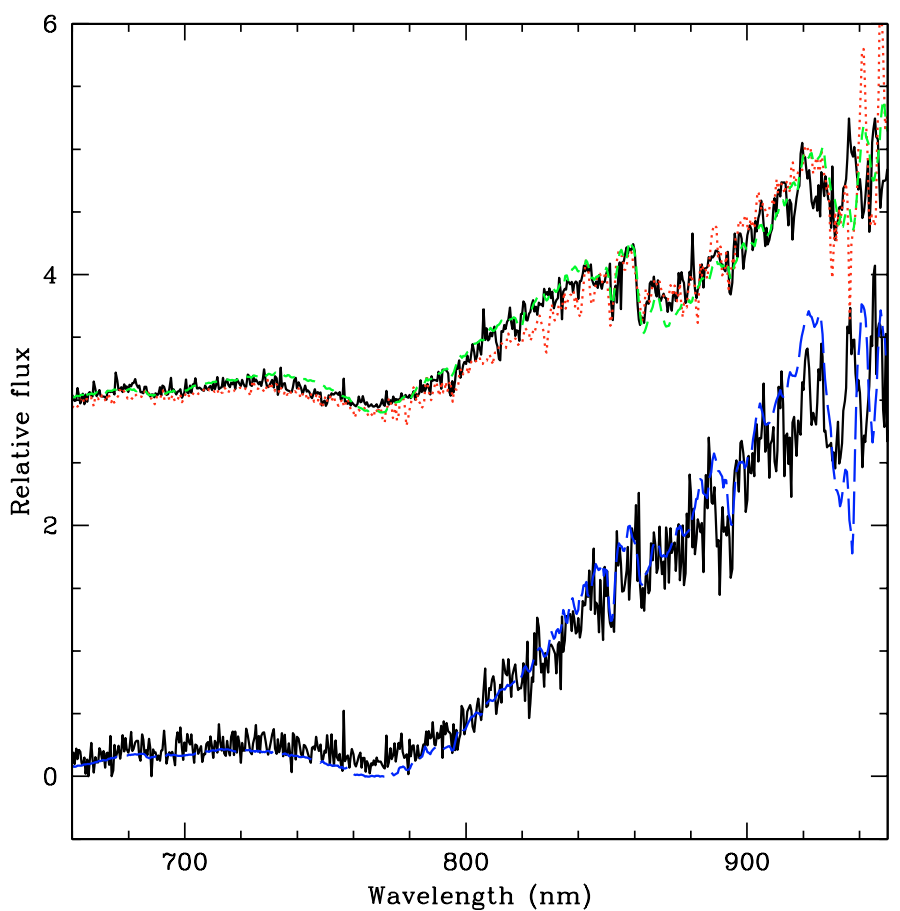

Fig. 3. Final STIS spectra of the components of the binary DENIS J0205-1159 A (top) and B (bottom). The following groundbased spectra of M99 are shown for comparison: DENIS J0205-1159 (dL5, red dotted line), DENIS J1228-1547 (bdL4.5, green short dashed line), and DENIS J0255-4700 (dL6, blue long-dashed line).

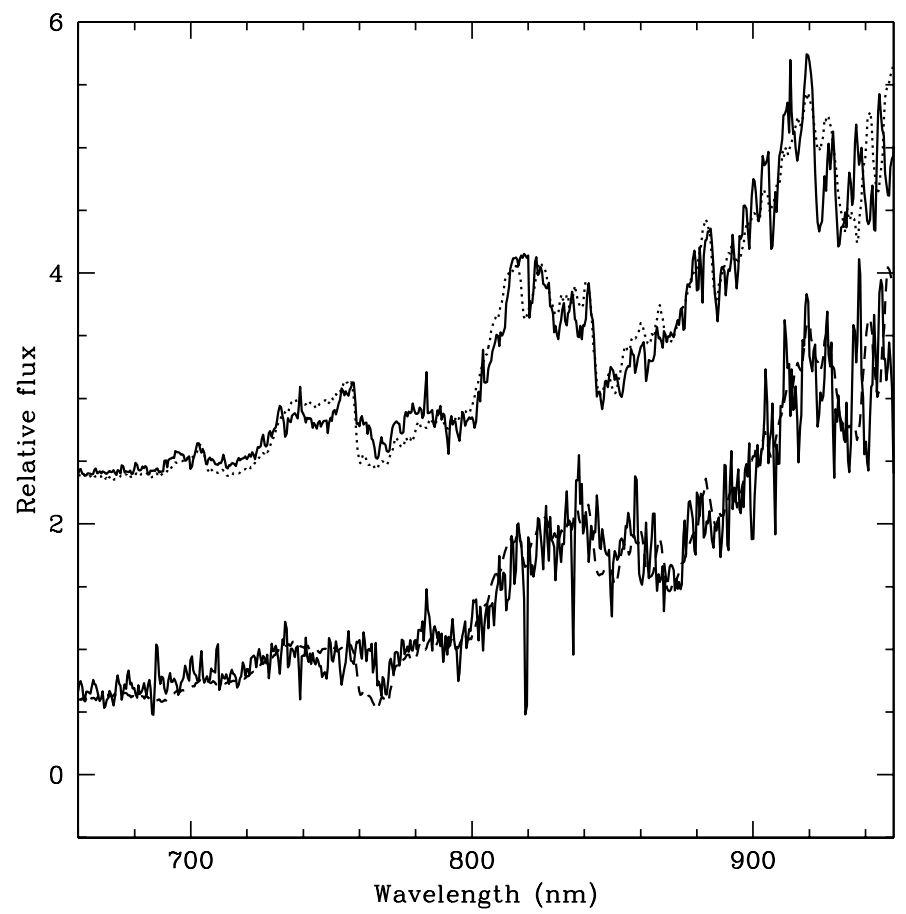

Fig. 4. Final STIS spectra of the components of the binary DENIS J0357-4417 A (top) and B (bottom). The following M99 spectra are shown for comparison: DENIS-P 1431-1953 (dM9, dotted) and DENIS J1441-0945 (dL1, dashed).

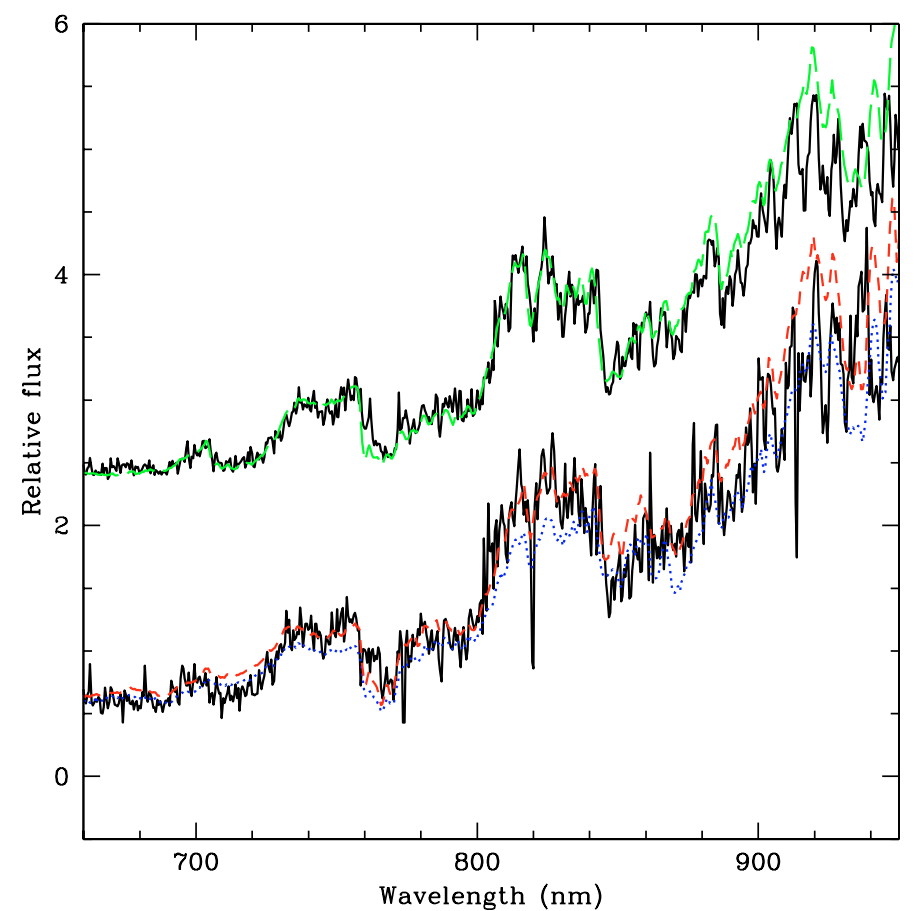

Fig. 5. Final STIS spectra of the components of the binary DENIS J1004-1146 A (top) and B (bottom). The following M99 spectra are shown for comparison: DENIS-P 1208+0149 (dM9.5, green longdashed line), DENIS-P 0909-0658 (dL0, red short-dashed line), and DENIS J1441-0945 (dL1, blue dotted line).

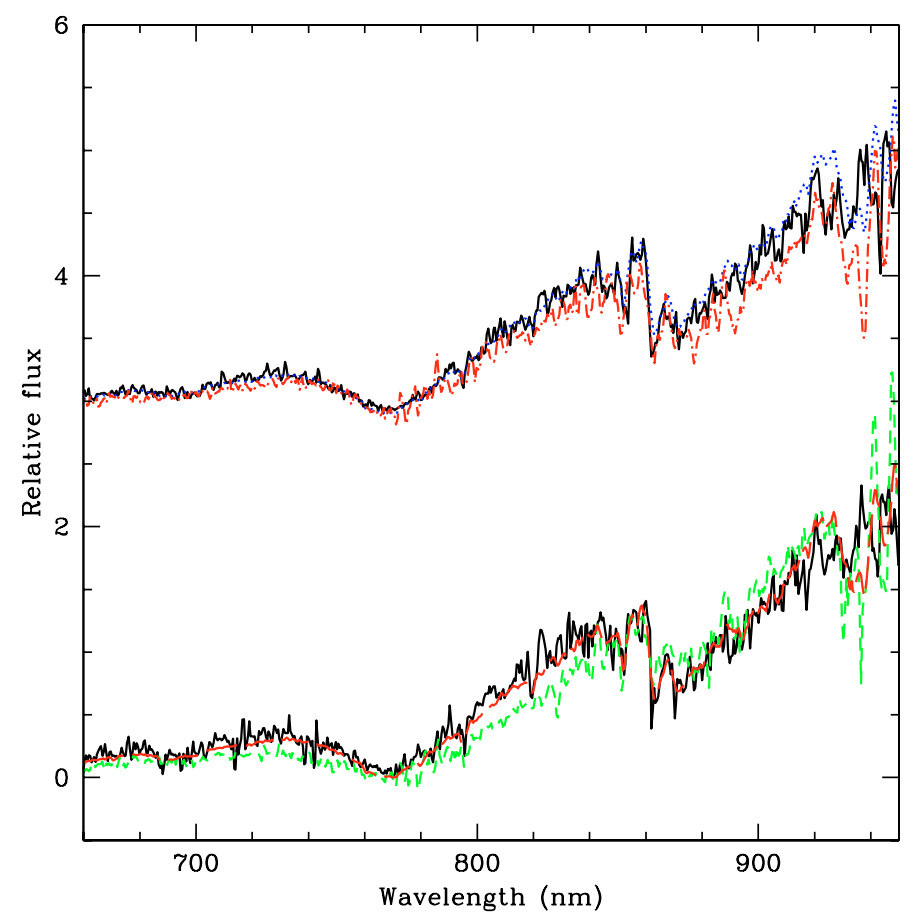

Fig. 6. Final STIS spectra of the components of the binary DENIS J1228-1547 A (top) and B (bottom). The following groundbased spectra of M99 are shown for comparison: LHS 102 B (dL4, red dot-short dash line on top part of the plot), DENIS J1228-1547 (bdL4.5, blue dotted line on top and red long-dashed line at the bottom), and DENIS J0205-1159 (dL5, green short-dashed line). 
E. L. Martín et al.: Resolved HST spectroscopy of ultracool binaries, Online Material p 6

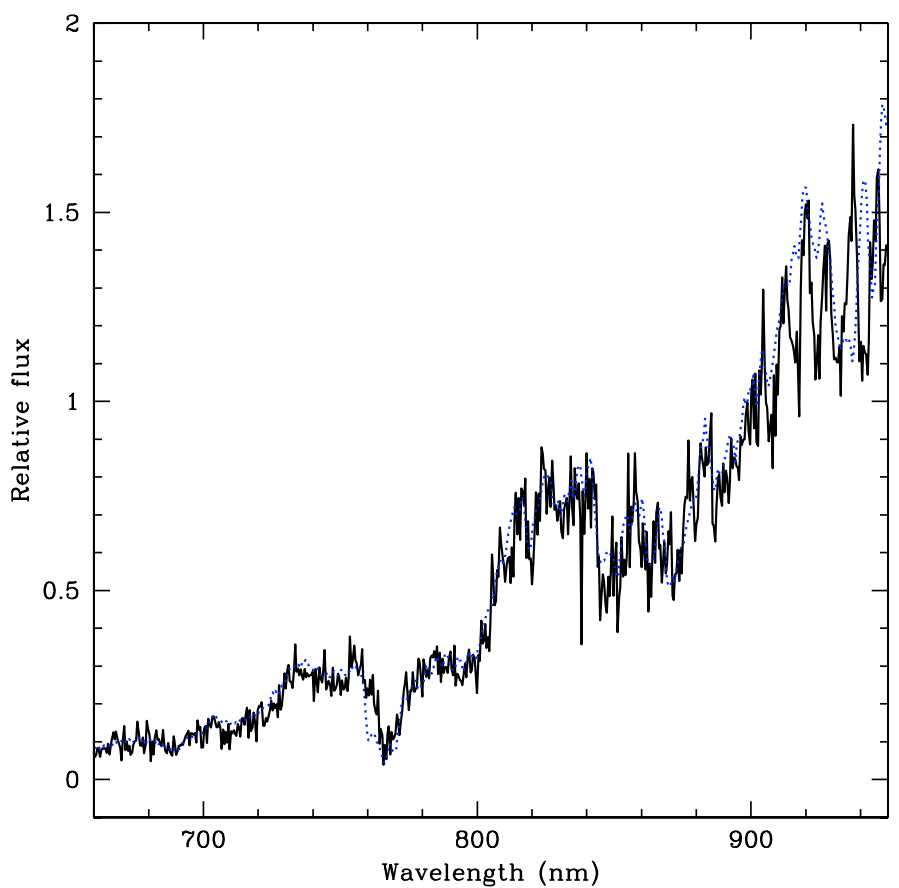

Fig. 7. Final STIS spectrum of DENIS J1441-0945 A compared with the M99 spectrum of DENIS J1441-0945 (dL1, blue dotted line).

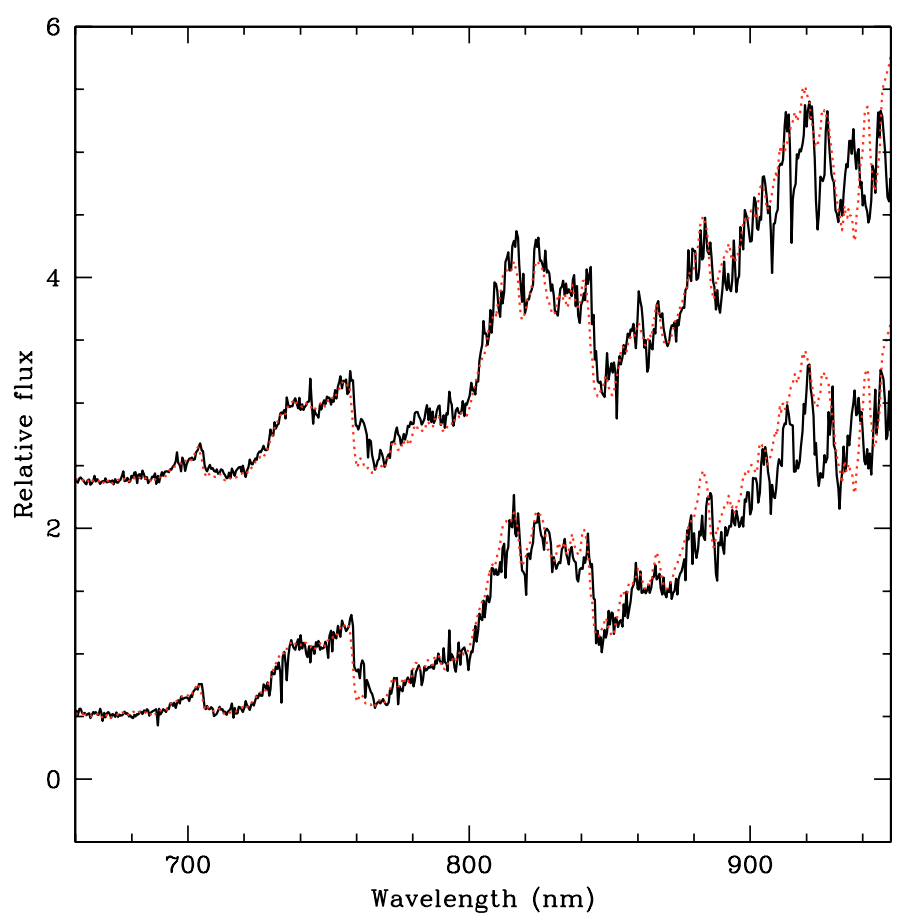

Fig. 8. Final STIS spectra of the components of the binary G1 $569 \mathrm{Ba}$ (top) and $\mathrm{Bb}$ (bottom), which are both matched with the M99 spectrum of DENIS-P 1431-1953 (dM9, red dotted line).

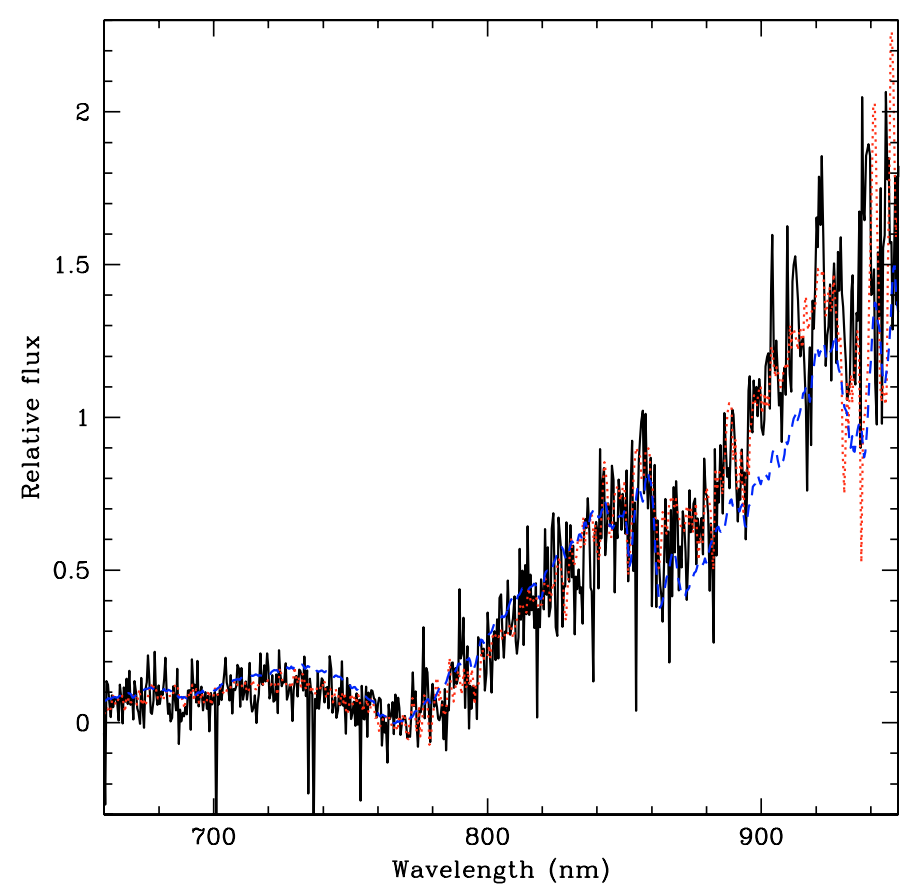

Fig. 9. Final STIS spectrum of 2MASS J0920+3517 A compared with the M99 spectra of DENIS J0205-1159 (dL5, red dotted line), and DENIS J1228-1547 (bdL4.5, dashed blue line).

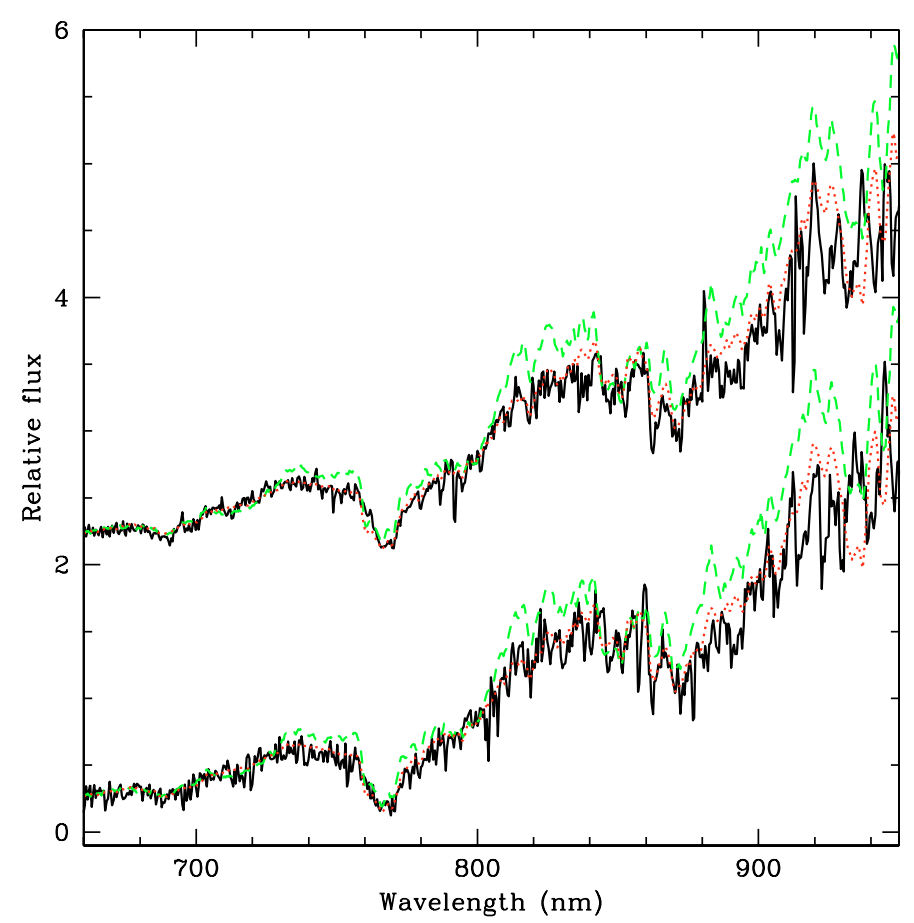

Fig. 10. Final STIS spectrum of 2 MASS J1146+2230 A (top) and B (bottom) compared with the M99 spectra of Kelu 1 (bdL2, red dotted line), and DENIS J1441-0945 (dL1, dashed green line). 
E. L. Martín et al.: Resolved HST spectroscopy of ultracool binaries, Online Material $p 7$

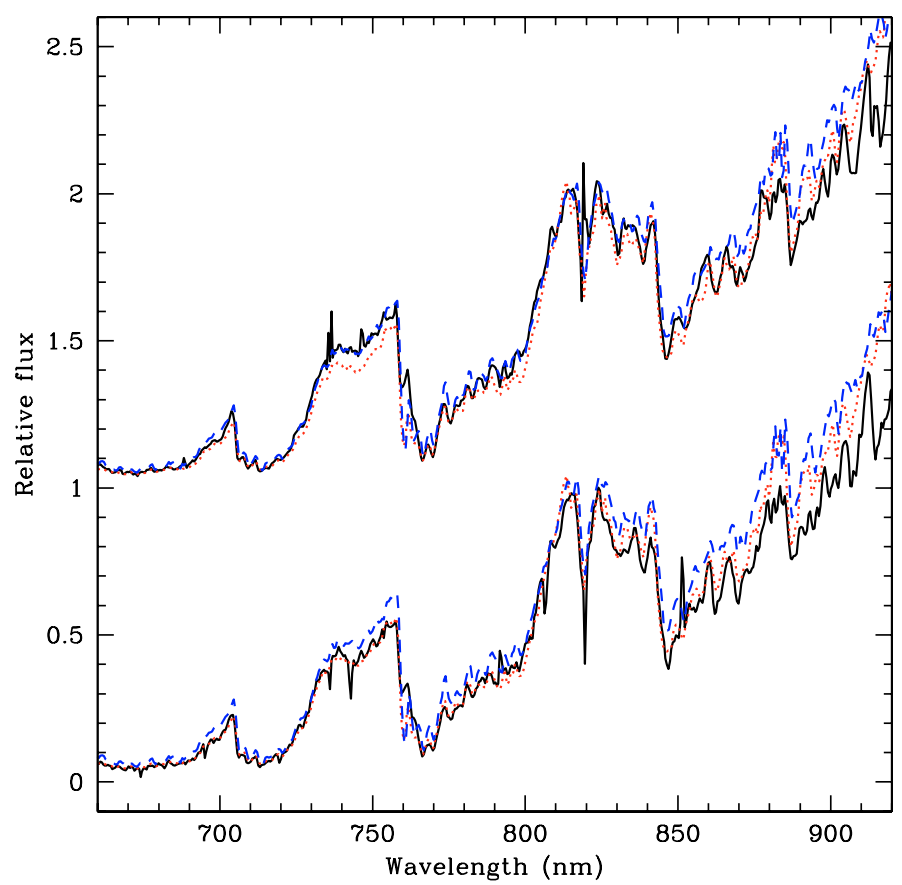

Fig. 11. Final STIS spectrum of 2 MASS J1311+8032 A (top) and B (bottom) compared with the M99 spectra of VB10 (dM8, red dotted line), and LHS2243 (dM7.5, dashed blue line)

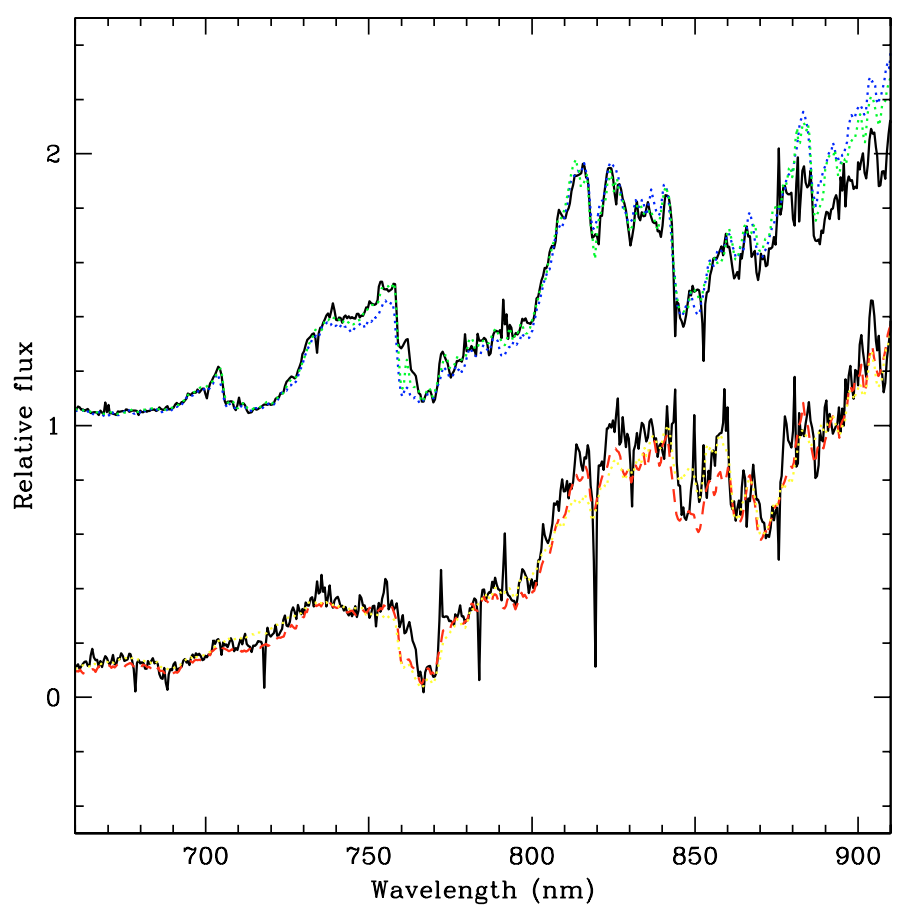

Fig. 12. Final STIS spectrum of 2 MASS J1426+1557 A (top) and B (bottom) compared with the M99 spectra of VB10 (dM8, green dotted line), DENIS-P 1431-1953 (dM9, blue dotted line), DENIS J1441-0945 (dL1, dashed red line) and Kelu 1 (bdL2, yellow dotted line) 